\begin{tabular}{|c|c|c|}
\hline $\begin{array}{r}\text { PRAMANA } \\
\text { - journal of } \\
\text { physics }\end{array}$ & (C) Indian Academy of Sciences & $\begin{array}{l}\text { Vol. } 79, \text { No. } 4 \\
\text { October } 2012 \\
\text { pp. } 643-658\end{array}$ \\
\hline
\end{tabular}

\title{
Top production at hadron colliders
}

\author{
ALBERT DE ROECK ${ }^{1,2,3}$ \\ ${ }^{1}$ CERN, 1211 Geneva 23, Switzerland \\ ${ }^{2}$ University of California-Davis, Davis, USA \\ ${ }^{3}$ Antwerp University, Antwerp, Belgium \\ E-mail: deroeck@mail.cern.ch
}

\begin{abstract}
New results on top quark production are presented from four hadron collider experiments: CDF and D0 at the Tevatron, and ATLAS and CMS at the LHC. Cross-sections for single top and top pair production are discussed, as well as results on the top-antitop production asymmetry and searches for new physics including top quarks. The results are based on data samples of up to $5.4 \mathrm{fb}^{-1}$ for the Tevatron experiments and $1.1 \mathrm{fb}^{-1}$ for the LHC experiments.
\end{abstract}

Keywords. Large Hadron Collider physics; top quarks; quantum chromodynamics; searches.

PACS Nos 13.85. $-t$; 14.65.Ha; 14.80.-j

\section{Introduction}

Top quark studies are an important aspect of physics program at the Tevatron and the LHC. Many new top quark results were produced since the Lepton-Photon Conference 2 years ago. In this review we discuss the top production properties in proton-antiproton collisions at $\sqrt{s}=1.96 \mathrm{TeV}$ and proton-proton collisions at $\sqrt{s}=7 \mathrm{TeV}$. The top quark is the heaviest known elementary particle with a mass of about $173 \mathrm{GeV}$ and a short lifetime of about $5 \cdot 10^{-25} \mathrm{~s}$, smaller than the typical hadronization time. The coupling to the Higgs boson is close to unity and hence it is generally thought that it may play a special role in EWK symmetry breaking mechanism, and possibly be directly connected to new physics. It thus offers a tool for searches for new physics. In this review we discuss top pair production at the Tevatron and the LHC, as well as single top production, and review the present precision with which we know the cross-sections. Recently, the Tevatron also reported the forward-backward asymmetries observed in top production which appear to be significantly larger than expected from NLO calculations. This could be a sign for new physics, when confirmed, and if it survives the addition of data and theoretical QCD developments. Also the LHC may be a referee with more data and improved control of the systematics using a similar measurement. Further, we also present searches for $t \bar{t}$ resonances, and for top partners and fourth-generation quarks in hadron-hadron collisions. 


\section{Albert De Roeck}

Top quarks were discovered in 1995 at the Tevatron, FNAL, US, by the CDF and D0 experiments [1,2]. Since then, the study of top quarks has been the playground of these experiments. It took however 14 more years to see the first convincing evidence for the more difficult channel of electroweak single top quark production [3,4]. End of March 2010 the LHC started its operation, producing $p p$ collisions at $7 \mathrm{TeV}$ CM system energy, and after a few months of running, with a statistics of only a few hundred $\mathrm{nb}^{-1}$ accumulated by the summer of 2010, the first top quark event candidates were observed and reported $[5,6]$. Within a year after the observation, the LHC produced a number of significant top measurements such as the top quark production cross-section and observation of the $t$-channel single top production process. By now (summer 2011 with $\sim 1-2 \mathrm{fb}^{-1}$ ) the LHC has about 10 to 20 times more top quark pairs for analysis than at the Tevatron, owing in large part to the large cross-section at the higher energy.

While at hadron colliders top quarks are essentially pair produced via the strong interaction, the main production channel for top production at the LHC is different from the one at the Tevatron. At the Tevatron the dominant production channel is quark-antiquark annihilation ( $\sim 85 \%$ of the cross-section) while at the LHC the dominant production mechanism is the gluon-gluon channel ( $\sim 85 \%$ of the cross-section). The latest predicted top quark cross-sections at the Tevatron and LHC are respectively

$$
\begin{aligned}
& \sqrt{s}=1.96 \mathrm{TeV}: \sigma(p p \rightarrow t \bar{t})_{\mathrm{NNLOapprox}}=7.46_{-0.67}^{+0.48} \mathrm{pb} \\
& \sqrt{s}=7 \mathrm{TeV}: \sigma(p p \rightarrow t \bar{t})_{\mathrm{NNLOapprox}}=164.6_{-15.7}^{+11.4} \mathrm{pb}
\end{aligned}
$$

These calculations are based on approximate NNLO evaluations [7,8]. One can see that the cross-section is about 20 times larger at the LHC compared to the Tevatron.

\section{Top cross-section}

The top quark events are typically divided into a number of different categories, depending on the decay mode of $W$ in the $t \rightarrow W b$ decay. The categories are defined according to the $W$ decaying leptonically (into an electron or a muon plus a neutrino) or hadronically. We thus have a dilepton channel ( $\sim 5 \%$ of the decays), a single lepton plus jets channel ( $\sim 30 \%$ of the decays) and the all hadronic multijet channel ( $\sim 46 \%$ of the decays). The remaining part of the decay modes is covered by channels where one or both $W$ bosons decay into a tau lepton and neutrino, but these are usually analysed separately, and yield generally less precise measurements. Top quark analysis techniques developed at the Tevatron include the use of likelihood functions, matrix elements, neural networks, boosted decision trees, and more, and are now also used and further refined at the LHC. Backgrounds are mostly estimated from data itself, e.g., multijets, $W+$ jets, Drell-Yan backgrounds, fake leptons, etc. Moreover, often $b$-quark identification is used to enhance the signal-to-background ratio. In all, top quark analyses require the understanding from essentially all physics objects at a collider experiment. The fact that the LHC experiments have entered the top quark study area so fast and swiftly was not obvious prior to data taking. 


\section{Top quark production}

\subsection{Recent Tevatron results}

D0 recently produced a new result based on $5.3 \mathrm{fb}^{-1}$ of data in the leptons plus jet channels. It combines the channels with 2, 3 or 4 and more jets, for both the electrons and muon channels, using one or more $b$-tags. The systematic limitations are the luminosity measurement, the jet energy scale and the systematics on the $b$-tagging. A precision on the cross-section of about $9 \%$ is reached. The result is $\sigma(p p \rightarrow t \bar{t})=7.78_{-0.64}^{+0.77} \mathrm{pb}$ where the error includes statistics, systematics and luminosity [9]. Another recent D0 result is the dilepton channel cross-section, which includes $b$-tagging. This is a neural net-based analysis and the result is $\sigma(p p \rightarrow t \bar{t})=7.36_{-0.79}^{+0.90} \mathrm{pb}$, i.e., leading to a $11 \%$ precision on the cross-section. Combining both D0 measurements gives $\sigma(p p \rightarrow t \bar{t})=7.56_{-0.56}^{+0.63} \mathrm{pb}$ total error, i.e., an $8 \%$ precision [10].

CDF has a new result on a cross-section measurement using top decays into the taus, based on the hadronic tau + jets channel. A neural net is used to suppress the dominant QCD background. The final result is $\sigma(p p \rightarrow t \bar{t})=8.8 \pm 3.3$ (stat) \pm 2.7 (sys) pb [11], as expected much less precise than the lepton plus jets and dilepton measurements. CDF has such new measurements, however. The new lepton plus jets channel measurement uses the CDF electroweak $W$ plus heavy flavour production measurement in order to have a better control on that dominant background component. Note however that CDF does measure a somewhat anomalously high cross-section in this EWK channel. Furthermore, the data are normalized to the $Z$ boson production to circumvent luminosity systematics. Two analyses were performed, one with the neural net and the other with the tagged $b$-jets. The result for the $b$-tagged jet analysis is $\sigma(p p \rightarrow t \bar{t})=7.32 \pm 0.36($ stat $) \pm$ 0.59 (sys) $\pm 0.14\left(Z_{\mathrm{TH}}\right) \mathrm{pb}$ and for the one based on $\mathrm{NN}$ shape variables is $\sigma(p p \rightarrow$ $t \bar{t})=7.82 \pm 0.38$ (stat) \pm 0.37 (sys) $\pm 0.15\left(Z_{\mathrm{TH}}\right) \mathrm{pb}$, leading to a $7 \%$ precision. In the dilepton channel, two recent analyses give $\sigma(p p \rightarrow t \bar{t})=7.40 \pm 0.58$ (stat) \pm 0.63 (sys) \pm 0.45 (lumi) pb and $\sigma(p p \rightarrow t \bar{t})=7.25 \pm 0.66$ (stat) \pm 0.47 (sys) \pm 0.44 (lumi) pb for the $b$-tagged analysis [12]. For better signal-to-background discrimination either the summed transverse energy $H_{\mathrm{T}}$ and missing $E_{\mathrm{T}}$ cuts were used, or $b$-tagging was required. The combined result from CDF based on a data sample including up to $4.6 \mathrm{fb}^{-1}$ of data is $\sigma(p p \rightarrow t \bar{t})=7.5 \pm 0.31$ (stat) \pm 0.34 (sys) \pm 0.15 (th) pb, i.e., $6.5 \%$ precision.

The Tevatron summary is shown in figure 1 for both CDF and D0, for $m_{\mathrm{t}}=172.5 \mathrm{GeV}$.

\subsection{Results from the $\mathrm{LHC}$}

New results from the ATLAS Collaboration for this summer on top cross-section measurement at $7 \mathrm{TeV}$ include the dilepton channel, based on $0.70 \mathrm{fb}^{-1}$ of the 2011 data. Two complementary analyses were performed with and without $b$-tagging. It is basically a cut and count method, using $H_{\mathrm{T}}$ and missing $E_{\mathrm{T}}$ cuts. The combined result of the two methods gives $\sigma(p p \rightarrow t \bar{t})=177 \pm 6(\text { stat })_{-14}^{+16}$ (sys) ${ }_{-7}^{+8}$ (lumi) pb, i.e., $11 \%$ precision [13].

CMS presented a new result this summer in the all hadronic channel, based on $1.1 \mathrm{fb}^{-1}$ of the 2011 data. The top cross-section is derived using an unbinned maximum likelihood to the reconstructed top mass peak, requiring two $b$-tagged jets. The QCD background is estimated from (antitagged) data. The result is $\sigma(p p \rightarrow t \bar{t})=$ $136 \pm 20$ (stat) \pm 40 (sys) \pm 8 (lumi) pb [14]. CMS also presented a dilepton analysis using the muon-tau channel, based on the hadronic decay of the tau, also based on 


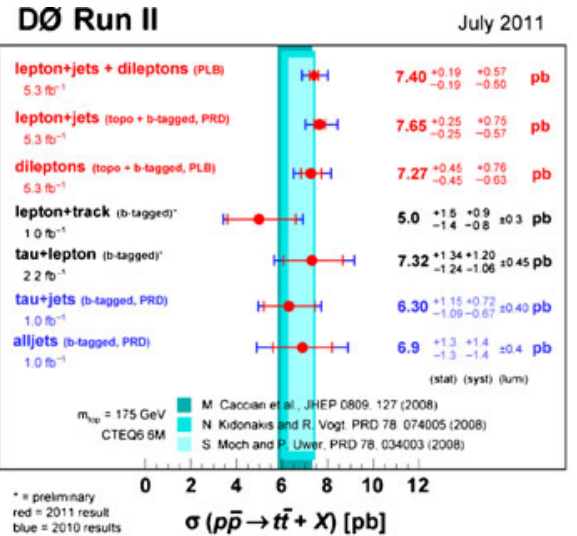

(a)

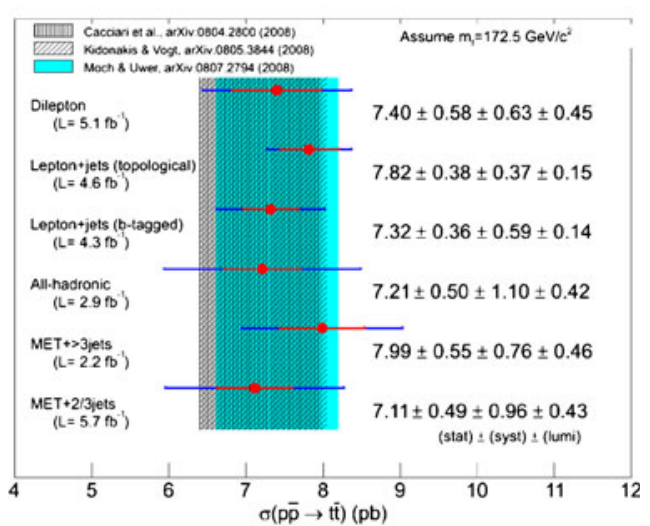

(b)

Figure 1. A summary of (a) the D0 and (b) CDF cross-section measurements.

$1.1 \mathrm{fb}^{-1}$ of the 2011 data. Such an analysis is also potentially sensitive to a light Higgs which would lead to an anomalously high tau measurement. The result is $\sigma(p p \rightarrow t \bar{t})=$ $148.7 \pm 23.6$ (stat) \pm 26.0 (sys) \pm 8.9 (lumi) pb [14].

Both experiments have released a combined measurement of their results, made available in the EPS Conference (July 2011). For ATLAS and CMS we get respectively $\sigma(p p \rightarrow t \bar{t})=176 \pm 5$ (stat) ${ }_{-10}^{+13}$ (sys) \pm 7 (lumi) $\mathrm{pb}$ and $\sigma(p p \rightarrow t \bar{t})=158 \pm 10$ (uncor.) \pm 15 (cor.) \pm 6 (lumi) pb, i.e., $9 \%$ precision. The results are in good agreement with the theoretical predictions. These results are shown, with the summer results of the Tevatron, in figure 2 .

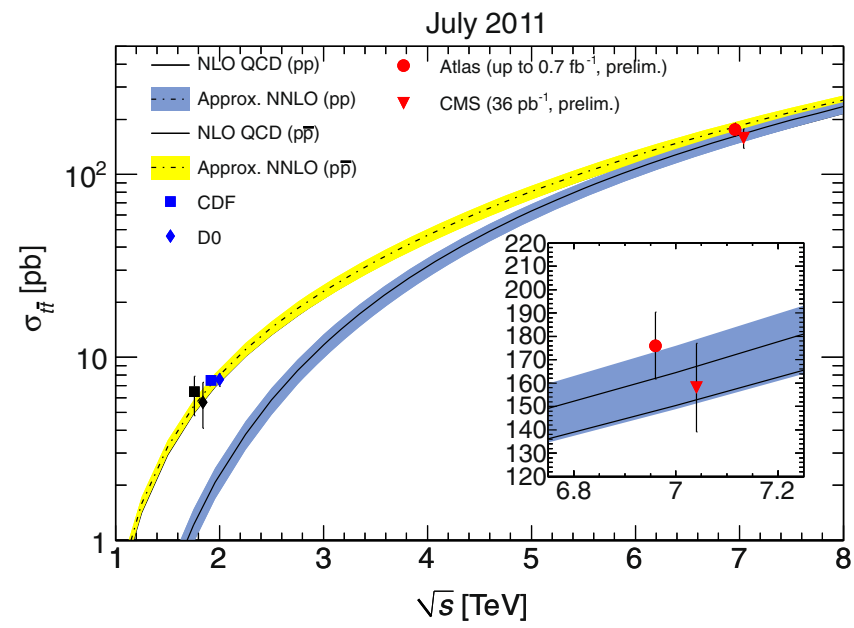

Figure 2. Summary of the $t \bar{t}$ cross-section measurements at the Tevatron and the LHC as a function of the centre of mass, energy as presented in July 2011. 


\section{Top quark production}

Table 1. Summary of the latest combined $t \bar{t}$ cross-section measurements for $m_{\mathrm{t}}=$ $172.5 \mathrm{GeV}$.

$$
\begin{array}{ll}
\text { CDF (up to } \left.4.6 \mathrm{fb}^{-1}\right) & \sigma(p \bar{p} \rightarrow t \bar{t})=7.5 \pm 0.31 \text { (stat) } \pm 0.34 \text { (syst) } \pm 0.15 \text { (th) } \mathrm{pb} \\
\text { D0 }\left(5.4 \mathrm{fb}^{-1}\right) & \sigma(p \bar{p} \rightarrow t \bar{t})=7.56_{-0.56}^{+0.63} \text { (stat }+ \text { syst }+ \text { lumi) pb } \\
\text { ATLAS (up to } \left.0.7 \mathrm{fb}^{-1}\right) & \sigma(p p \rightarrow t \bar{t})=176 \pm 5 \text { (stat) }{ }_{-10}^{+13} \text { (syst) } \pm 7 \text { (lumi) pb } \\
\text { CMS }\left(36 \mathrm{pb}^{-1}\right) & \sigma(p p \rightarrow t \bar{t})=158 \pm 10 \text { (uncor.) } \pm 15 \text { (cor.) } \pm 6 \text { (lumi) pb }
\end{array}
$$

The results of the combined values before LP11 are given in table 1 [15]. Many new results were shown for the first time at the LP11 Conference which will be discussed next.

ATLAS released a dilepton muon plus tau, plus two jets analysis, of which at least one jet is $b$-tagged. A boosted decision tree method was used for this analysis, and $1.08 \mathrm{fb}^{-1}$ of data was included. The result of the measurement is $\sigma(p p \rightarrow t \bar{t})=$ $142 \pm 21$ (stat) ${ }_{-16}^{+20}$ (sys) \pm 5 (lumi) pb [16]. Probably the most precise LHC measurement to date at this conference comes from the ATLAS leptons plus jets measurement, including both muons and electron channels, which makes use of the kinematicls differences between the $t \bar{t}$ and the $W+$ jets events. No $b$-tagging is used for this analysis. Several categories of jets number classes are analysed individually. Figure 3 shows the results of the combined fit to data in the six analysis bins. Based on $0.70 \mathrm{fb}^{-1}$, ATLAS reports $\sigma(p p \rightarrow t \bar{t})=179.0 \pm 3.9$ (stat) \pm 9.0 (sys) \pm 6.6 (lumi) pb, i.e., $7 \%$ precision [17].

For LP11 CMS released a dilepton analysis, using muons and electrons, and having two $b$-tagged jets, based on $1.1 \mathrm{fb}^{-1}$, using a cut and count method. The result is $\sigma(p p \rightarrow$ $t \bar{t})=169.9 \pm 3.9$ (stat) \pm 16.3 (sys) \pm 7.6 (lumi) $\mathrm{pb}[18]$.

The overall LP11 summary of results from the LHC is shown in figure 4. The most precise single value is now $7 \%$, driven by the understanding of the systematics and the

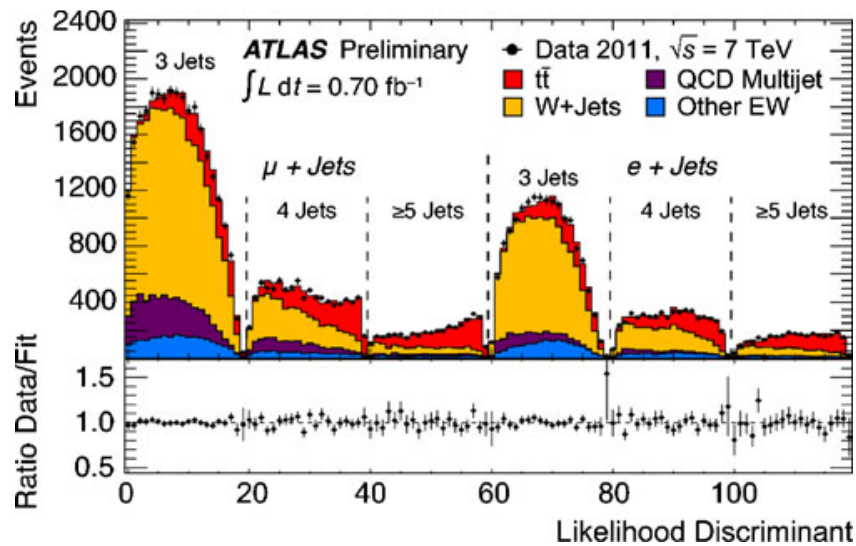

Figure 3. Result of combined fit to data in the exclusive three-jet bin, the exclusive four-jet bin and the inclusive five-jet bin of the electron + jets and muon + jets channels. The lower plot shows the ratio of the data to the sum of fitted signal and background contributions. Uncertainties on the ratio include data and MC statistical uncertainties. 


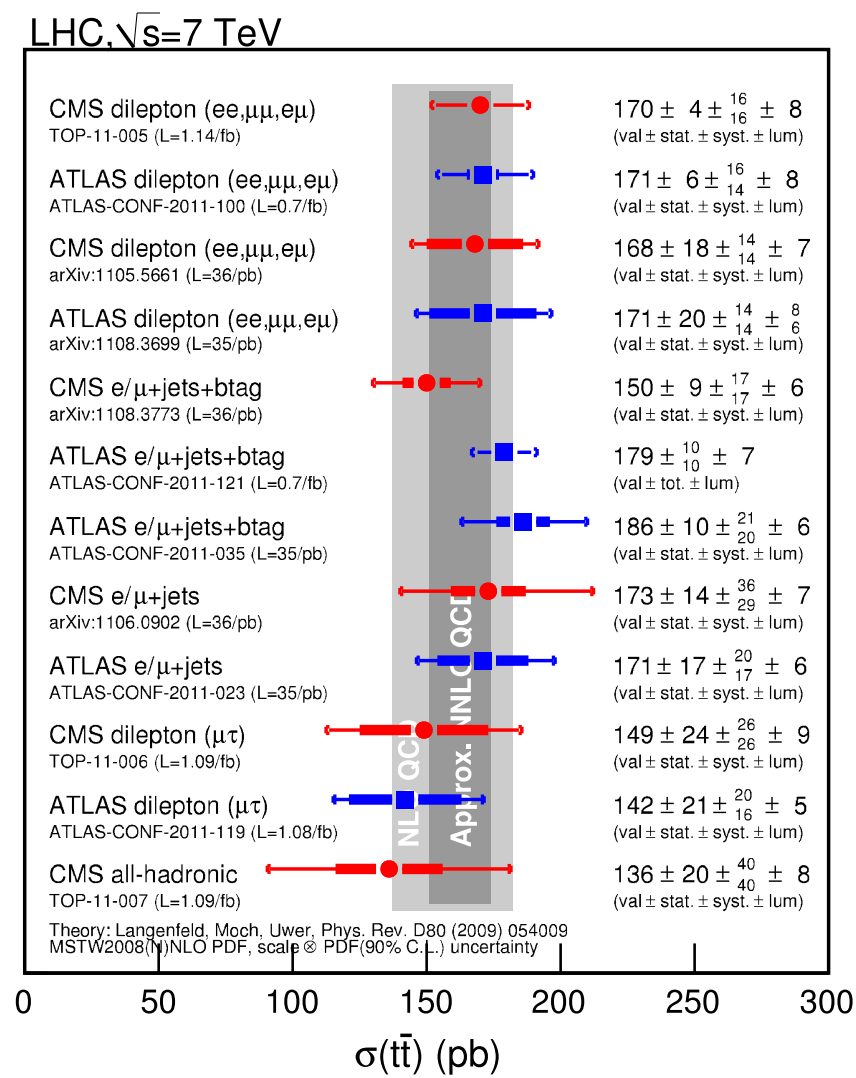

Figure 4. An overview of the ATLAS and CMS top pair production cross-sections released by the time of LP11 measurement.

luminosity. In the figure we see that the precision of the NNLO approximation is now similar to the experimental precision and will be challenged soon. This is also the case for the Tevatron measurements and hence more precise calculations are called for. There is also a plan to set up a LHC-wide combinations group in fall 2011 to combine the crosssection results from ATLAS and CMS. One could expect perhaps a precision of about $5 \%$ to be reached soon when the luminosity error can be reduced or other normalization methods are used.

\subsection{Further impact of the top quark cross-section results}

Top quark cross-sections can be used further to constrain parton distribution functions (PDFs). This is illustrated in figure 5 where the HATHOR [8] program was used to calculate NLO and NNLO(approx) cross-sections for different PDFs with their uncertainties, and which are compared to the present single most precise measurement at the LHC [19]. One can see that the top cross-section measurements already start to discriminate among 


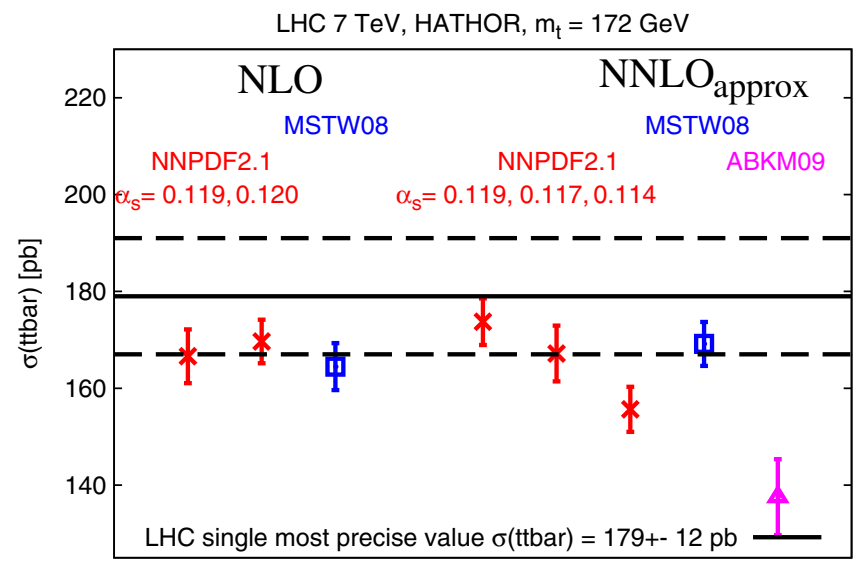

Figure 5. Various predictions of the top pair production cross-section for different PDFs, at NLO (approx).

the PDFs. More such comparisons are also shown in [20,21]. In future one can also study e.g., $t \bar{t}$ to $Z$ ratios, which are anticorrelated in the PDFs. In all, top cross-section measurements are reaching a precision which will make them a useful new ingredient for PDF fits in the near future.

A precise measurement of the top cross-section can be used to give information on the top quark mass. The relative uncertainty on the cross-section translates to an approximate five times smaller relative uncertainty on the mass, e.g., $10 \% \rightarrow 2 \%$. Inclusive crosssection calculations as functions of the top mass are available for different mass schemes.

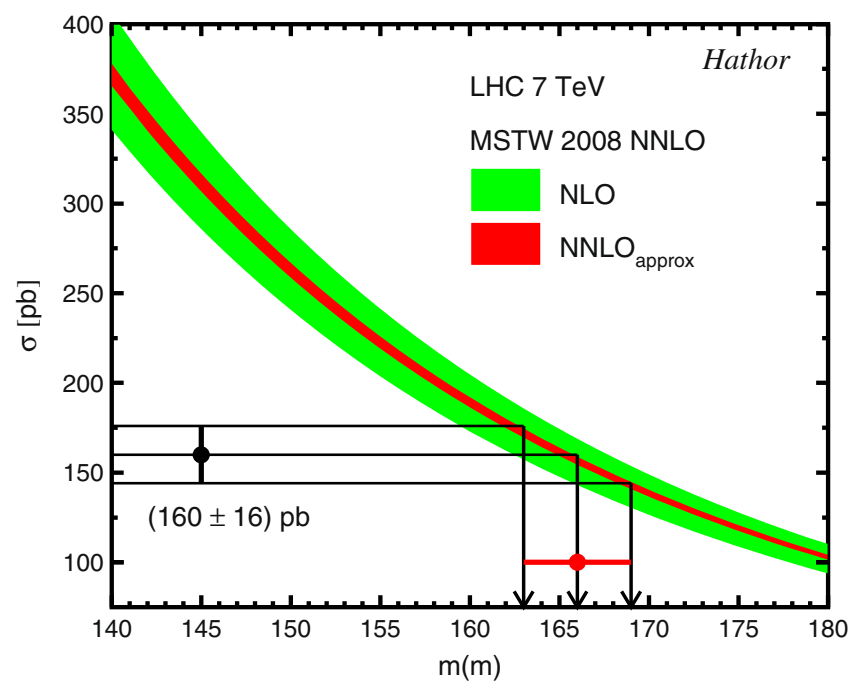

Figure 6. Relation between the top quark mass and top cross-section, and projected uncertainties. 


\section{Albert De Roeck}

Hence, such a measurement also forms a good test for differences in the different mass measurement schemes (pole, $\overline{\mathrm{MS}}$ ). Figure 6 shows how a relative uncertainty on the cross-section translates to an uncertainty for the top mass, as function of the mass of the top quark, calculated by [22]. D0 and ATLAS have recently shown results using this technique $[23,24]$. D0 showed that the directly measured 'MC' mass from the top decays used by most mass determination techniques is indeed close to the pole mass (as compared to the $\overline{\mathrm{MS}}$ mass). For ATLAS the present uncertainties on the mass measured via this method, based on $35 \mathrm{pb}^{-1}$ of data, amount to about $5 \mathrm{GeV}$. A mass determination with a precision of about $1-2 \%$ seems within reach with future top cross-section measurements.

\section{Single top production}

While the dominant production mechanism for top quarks at hadron colliders is the strong interaction, another interesting channel is the electroweak production of single top. Single top quark production at hadron colliders was first established in 2007 by the Tevatron experiments. Basically, there are three production processes: the $s$-channel, the $t$-channel and the $W t$-channel. From these three processes the $t W$ process is not accesible at Tevatron and the $s$-channel process is very challenging at LHC. Single top production is also a way to measure directly the $V_{t b}$ matrix element, e.g., to constrain the $b$-quark PDFs in the proton. Furthermore, it is anticipated to be strongly affected by many new physics scenarios, manifesting themselves through anomalous couplings. So confronting the measurement with Standard Model predictions is mandatory. Single top production measurements are a challenge however. One has to deal with small cross-sections and backgrounds that are very similar to the signal.

At the LHC the observation of single top production came already within the first year of operation, which was surely surprising, given that only $35 \mathrm{pb}^{-1}$ of data was available and the first sighting of the top quarks was done only six months before.

The LHC experiments have established and measured single top production in $p p$ collisions at $7 \mathrm{TeV}$ in the $t$-channel. The expected cross-section is about $65 \mathrm{pb}$. The selection has been optimized particularly for the $t$-channel study, with cut-based and multivariate analyses. Generally the lepton plus jets ( $b$-tagging for one jet) channel is used, including missing $E_{\mathrm{T}}$ also. The results for both experiments are $\sigma(p p \rightarrow t)=90_{-9}^{+9}(\mathrm{stat})_{-20}^{+31}(\mathrm{sys})$ $\mathrm{pb}\left(\right.$ ATLAS, $\left.0.7 \mathrm{fb}^{-1}\right)[28]$ and $\sigma(p p \rightarrow t)=83.6 \pm 29.8$ (stat + sys) \pm 3.3 (lumi) pb $\left(\mathrm{CMS}, 35 \mathrm{pb}^{-1}\right)$ [27].

Recent analyses of CDF and D0 perform at simultaneous determination of $s$ - and $t$-channel contributions, using MVA techniques. CDF and D0 train the MVA differently, and get somewhat different results with respect to the significance of the measurement in the two channels. Figure 7 shows the results of a model-independent measurement of $t$ - and $s$-channel single top quark production [25]. D0 also has new measurements of $0.79<\left|V_{t b}\right|<1.0$ at $95 \%$ CL [26].

The summary of the $t$-channel measurements at the LHC and Tevatron is shown in figure 8. The values for the Tevatron experiments, extracted for a top mass of $172.5 \mathrm{GeV}$, are $\sigma(p p \rightarrow t)=0.8 \pm 0.4 \mathrm{pb}\left(\mathrm{CDF}, 3.2 \mathrm{fb}^{-1}\right)$ and $\sigma(p p \rightarrow t)=2.9 \pm 0.59 \mathrm{pb}(\mathrm{D} 0$, $\left.5.4 \mathrm{fb}^{-1}\right)$. The expected cross-section at the Tevatron is about $2.3 \mathrm{pb}$. 


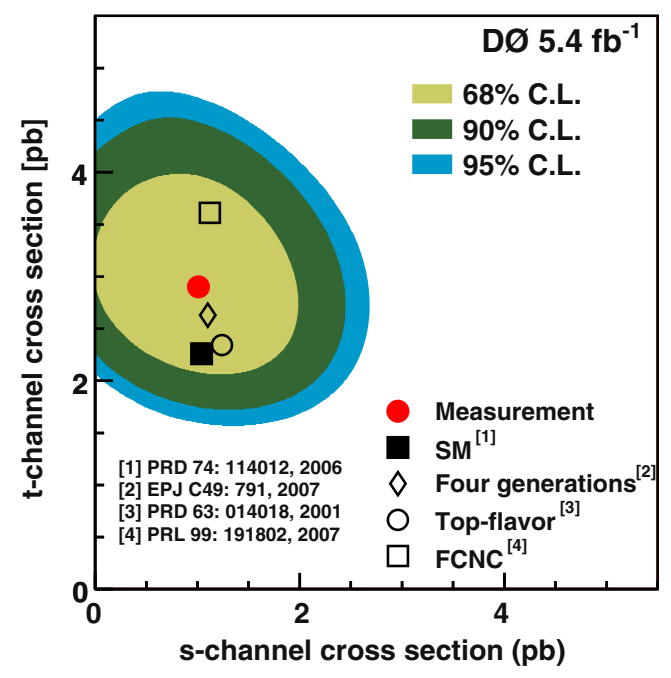

Figure 7. Posterior probability density for $t q b$ vs. $t b$ single top quark production in contours of equal probability density. The measured cross-section and various theoretical predictions are also shown.

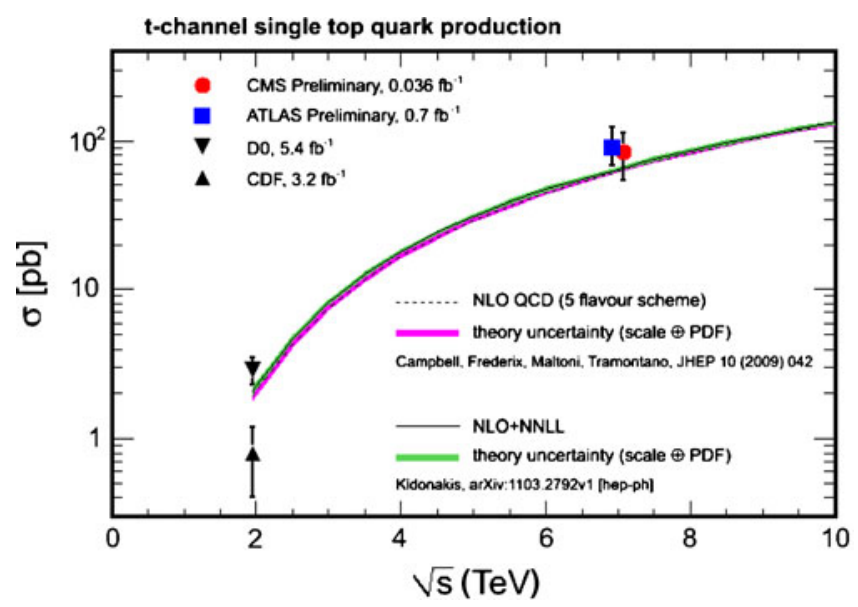

Figure 8. Single top $t$-channel cross-sections at the Tevatron and the LHC.

ATLAS has also released a search for the $s$-channel using a cut-based analysis, lepton + missing $E_{\mathrm{T}}$ and two $b$-tagged jets. The Standard Model expectation is about $5 \mathrm{pb}$. The data are not yet sufficiently sensitive for an observation of the Standard Model crosssection. Hence a $95 \%$ CL upper limit of $\sigma_{\mathrm{s}}<26.5 \mathrm{pb}$ is quoted [29]. It excludes scenarios with very large anomalous couplings at the LHC energies. Also, a search for the Wt channels was performed, with a cut-based analysis, using the leptonic $W$ decays. An observed 


\section{Albert De Roeck}

95\% CL upper limit of $\sigma_{\mathrm{Wt}}<39.1 \mathrm{pb}$ is produced [30]. The Standard Model expectation is $15.7 \mathrm{pb}$ for this channel. With the 2011 and 2012 statistics, the LHC experiments will have a plethora of studies to perform in the single top sector.

\section{Top quarks in searches for new physics}

Top quarks may well play a special role in Nature and connect to new physics perhaps more rapidly or strongly than the lighter quark species. It is therefore important that we keep confronting the observables in the top sector with Standard Model expectation.

\subsection{Forward-backward and charge asymmetry}

An interesting measurement discussed at this conference was the top production asymmetry measurement from the Tevatron. QCD predicts that top quark production is forward-backward symmetric at LO but at higher orders a positive asymmetry appears where the top is produced preferably in the direction of the initial quark in the collision, and correspondingly the antitop is produced with preference in the antiquark direction. At the Tevatron, being a proton-antiproton collider, this translates into a forward-backward asymmetry. This asymmetry has sensitivity to certain new physics processes. The interest in this measurement was generated by the result from CDF released, earlier this year, that showed a large deviation from theory than expected, and an apparent increasing deviation for larger invariant mass values of the top-antitop system [31]. The CDF result and theory prediction are shown in figure 9 . Inclusively, $\mathrm{CDF}$ measures $A_{\mathrm{FB}}=0.158 \pm 0.075$ while NLO theory predicts $0.058 \pm 0.009$, also discussed in the talk of Petriello at this conference.

Recently, new results from D0 [32] confirm the discrepancy, but no clear mass dependence is found, as shown in figure 10. In total the discrepancies now reported amount to 2.4 to $3 \sigma$ (the latter for the lepton-based asymmetry of D0). The results are shown in figure 10 for two mass bins. There is no clear mass dependence in the D0 data, as compared to the CDF data, but the data are still compatible at this stage. It will be interesting to see what the results will be with full Tevatron datasets of more than $10 \mathrm{fb}^{-1}$. D0 also found that there are some issues in describing the data with the Monte Carlo, and one should also note that the asymmetry only appears at NLO. So NLO calculations may not yet be a complete description for the effect. Hence, at present it cannot be excluded that the observed discrepancy may be an incomplete understanding of QCD.

The LHC experiments do not have such a forward-backward reference, the LHC being a $p p$ collider. However, the asymmetry should persist in the absolute value measurement of the (pseudo-) rapidity spectra. Indeed, in quark-initiated processes, there will be a quark-antiquark difference since the quarks have a large valence quark contribution while the antiquarks will come only from sea quarks with a different Bjorken- $x$ distribution and therefore rapidity distribution. Hence at the LHC we define the variable

$$
A_{C}=\frac{N(\Delta|y|>0)-N(\Delta|y|<0)}{N(\Delta|y|>0)+N(\Delta|y|<0)}
$$




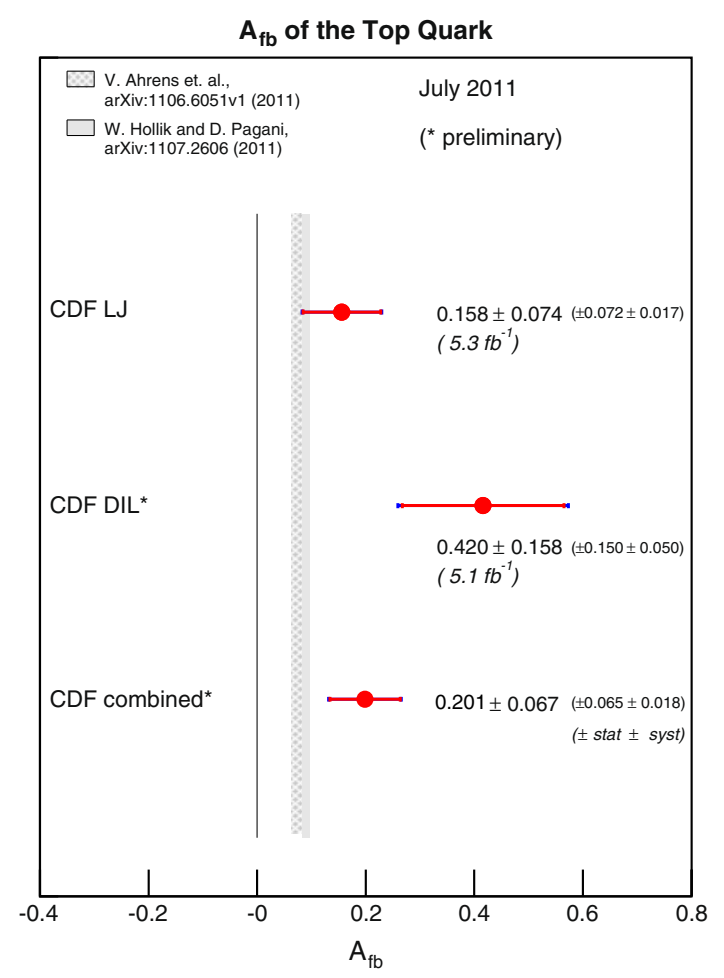

Figure 9. CDF results on the forward-backward asymmetry for different channels, compared with theory predictions.

with $\Delta|y|=\left|y_{\mathrm{t}}\right|-\left|y_{\mathrm{t}}\right|$ and similarly for pseudorapidity $\eta$. CMS [33] and ATLAS [34] have shown new measurements of $A_{C}$ with results with data samples of respectively $1.1 \mathrm{fb}^{-1}$ and $0.7 \mathrm{fb}^{-1}$ for CMS and ATLAS.

$\mathrm{CMS}: \quad A_{C}^{\eta}=-1.6 \pm 3.0(\mathrm{stat})_{-1.9}^{+1.0}(\mathrm{sys}) \%, \quad A_{C}^{\eta}(\mathrm{TH})=1.3 \%$,

ATLAS: $\quad A_{C}^{y}=-2.4 \pm 1.6($ stat $) \pm 2.3($ sys $) \%, \quad A_{C}^{y}(\mathrm{TH})=0.6 \%$.

The effects at the LHC are clearly smaller than at the Tevatron, mostly due to the dilution of the gluon-gluon dominant production process which does not contribute to the asymmetry. CMS also made a study of the effect as function of the top-antitop invariant mass, as shown in figure 11a, but does not see any striking mass dependence.

New physics scenarios have been proposed to explain this Tevatron 'anomaly' based on flavour changing neutral currents. This would lead for most models to same sign top production at LHC. CMS has made a recast of a published SUSY search in the like-sign lepton channel into a search for same sign top [35]. No evidence was found and the 
Forward-Backward Top Asymmetry, \%

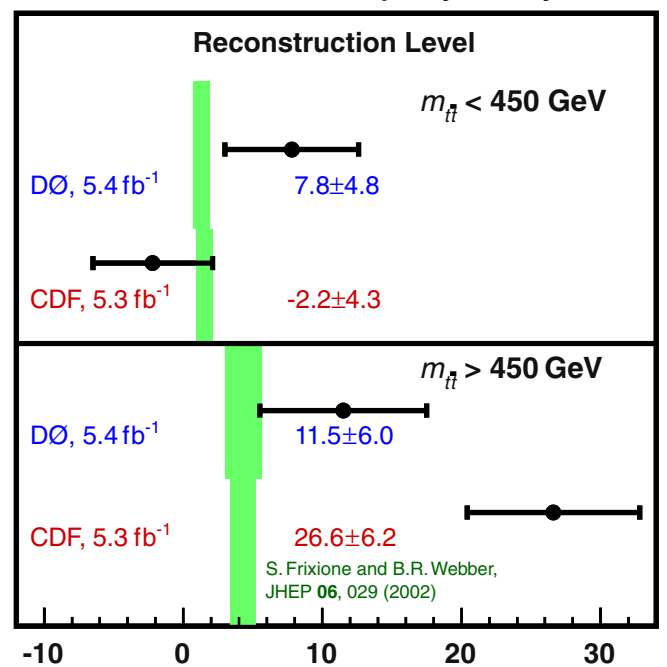

Figure 10. The forward-backward asymmetry as a function of the invariant mass of the top-antitop quark pair, for two mass bins.
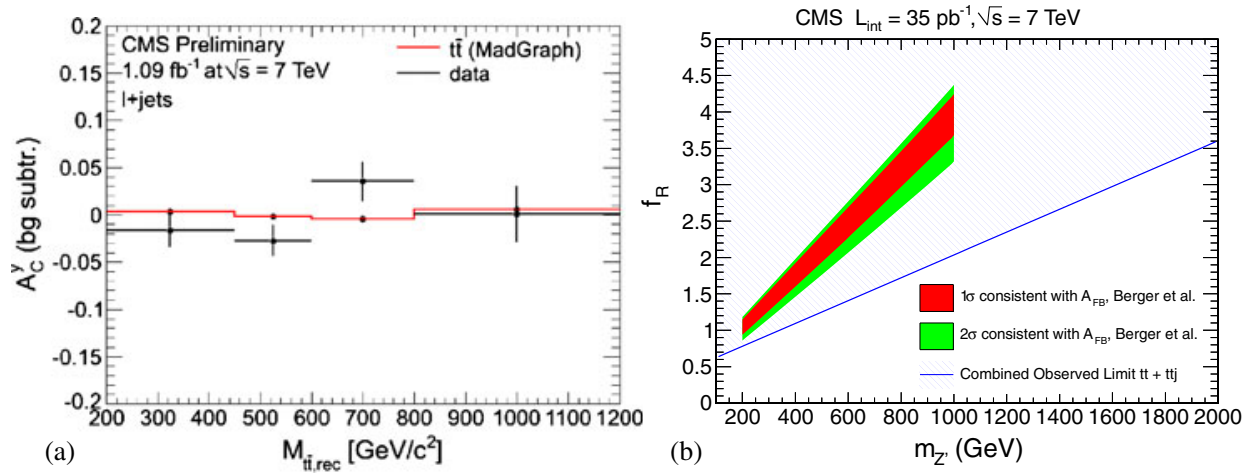

Figure 11. (a) The measurement of $A_{C}$ as a function of the invariant mass of the top-antitop quark pair, (b) the exclusion region at $95 \% \mathrm{CL}$ as a function of $Z$ mass for various choices of the right-handed coupling $f_{\mathrm{R}}$. We also show the region of parameter space consistent with the Tevatron measurements of $A_{\mathrm{FB}}$ and $\sigma(t \bar{t})$.

result has been cast into an exclusion limit shown in figure 11b. The region also excludes the 'sweet spot' for the simplest models compatible with the Tevatron large asymmetry measurement. Hence the search is still on, on all fronts, to clarify the situation. Ultimately, sufficient precision should be reached at the LHC to referee the situation, but probably the most urgent part is aiming for a deeper understanding in $\mathrm{PQCD}$ of this asymmetry. 


\section{Top quark production}

\subsection{Searches for a heavy $Z^{\prime}$ decaying into top quarks}

Given its special nature, the top quark plays a prominent role in many models for new physics. One example is top quarks in the decay of heavy objects, such as $Z^{\prime}$-like bosons. CDF [36] produced recently a new search result for a $Z^{\prime}$ decaying into a top-antitop quark pair, by inspecting the measured top-antitop invariant mass $M_{\mathrm{t} \mathrm{t}}$. No excess has been observed and a leptophobic heavy $Z$ is excluded with a mass lower than $900 \mathrm{GeV}$ at $95 \% \mathrm{CL}$, as shown in figure 12, assuming sequential model type of cross-sections.

The Tevatron reaches its kinematical limit for an efficient production of objects heavier than $1 \mathrm{TeV}$, and this is where the LHC takes over. Hence, similar searches have been carried out at the LHC [37-39] using the lepton plus jet decay channel, as well as the dilepton channel. With the present data samples, the LHC is now excluding typically a mass range up to and beyond $1 \mathrm{TeV}$, for different physics scenarios. Many of these analyses are based on conventional methods for the selection of events containing top quarks, much along the lines used so far in results reported in this review. However, for very massive $Z^{\prime}$-like objects, namely in the multi-TeV range, the decay of the top quarks will be highly boosted in the LHC rest frame. In the hadronic decays several jets will merge into one or two unusual fat jets. CMS released a special analysis in the search for highly boosted top quarks in the all hadronic decay channel for the top quarks [40]. The analysis starts from the Cambridge-Aachen jets finder, searching for fat jets, and applies jet pruning to find subjets. The major concern for this analysis is the QCD background estimate, which is taken from data, using a miss-tag method. CMS uses a particle flow technology to reconstruct the jets, which turned out to be a real asset for this study. The results based on $35 \mathrm{pb}^{-1}$ put limits on excluding the so-called $K K$-gluons in the mass range of $1<M<1.5 \mathrm{TeV}$.

Finally, a new ATLAS search for resonances decaying into top quarks was released [41]. This search uses lepton plus jets $t \bar{t}$ events and analyses the invariant mass distribution. A search is conducted for narrow topcolour $Z$ and also wider $K K$ gluons. With the present data, a limit on $K K$-gluons with mass lower than $700 \mathrm{GeV}$ at $95 \% \mathrm{CL}$ can be derived.

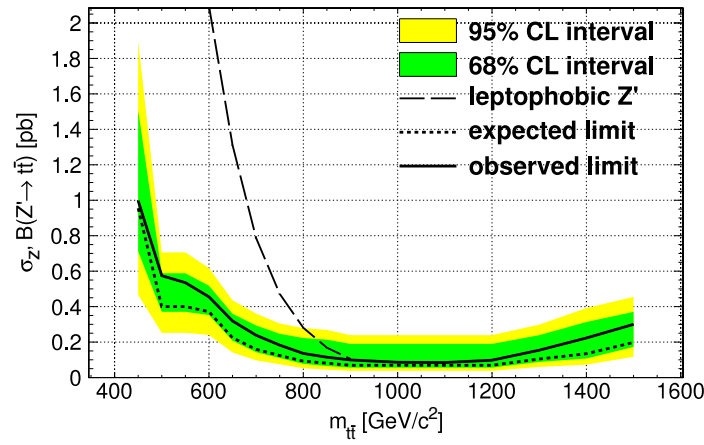

Figure 12. Expected and observed 95\% CL upper limit for $\sigma\left(p p \rightarrow Z^{\prime}\right) \rightarrow$ $\mathrm{BR}\left(Z^{\prime} \rightarrow t \bar{t}\right) 4.8 \mathrm{fb}^{-1}$ of integrated luminosity as a function of reconstructed $t \bar{t}$ invariant mass. The solid line indicates the observed limit. The dashed line indicates the theoretical cross-section for a leptophobic $Z^{\prime}$. 

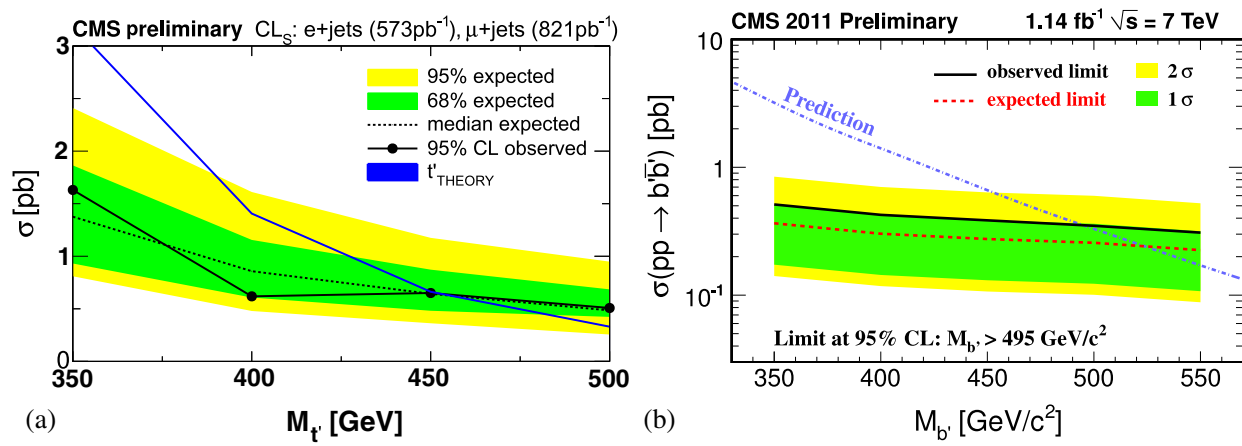

Figure 13. (a) Observed and expected limits for for $p p \rightarrow t t^{\prime \prime}$ pair production. (b) The exclusion limits at the $95 \% \mathrm{CL}$ on the $p p \rightarrow b^{\prime} b^{\prime}$ production cross-section. The solid line represents the observed limits, while the dotted line represents the limits expected with the available integrated luminosity, assuming the presence of Standard Model processes alone. Comparing with the production cross-sections, $b^{\prime}$ mass less than $495 \mathrm{GeV} / \mathrm{c}^{2}$ is excluded with an assumption of $100 \% b^{\prime} \rightarrow b W$ decay branching fraction.

\subsection{Search for $t^{\prime}, b^{\prime}$ and top partners}

The minimal fourth-generation models are presently not favoured by the recent Higgs exclusion limits, but the experiments have the duty to search for a new heavy generation or top like partners, e.g. as they can appear in little Higgs models.

Studies were conducted, e.g., in the channel $t^{\prime} t^{\prime} \rightarrow W b W b \rightarrow l v b q \bar{q} b$, and reported in [42-45]. No $t^{\prime}$ particles have been found for masses below $450 \mathrm{GeV}$ at $95 \%$ CL. Further searches include the channels $b^{\prime} b^{\prime} \rightarrow t W t W \rightarrow b W W b W W$ [46]. No $b^{\prime}$ quark with $t W$ decay is found with mass below $495 \mathrm{GeV}$ at $95 \% \mathrm{CL}$. The results are shown in figure 13.

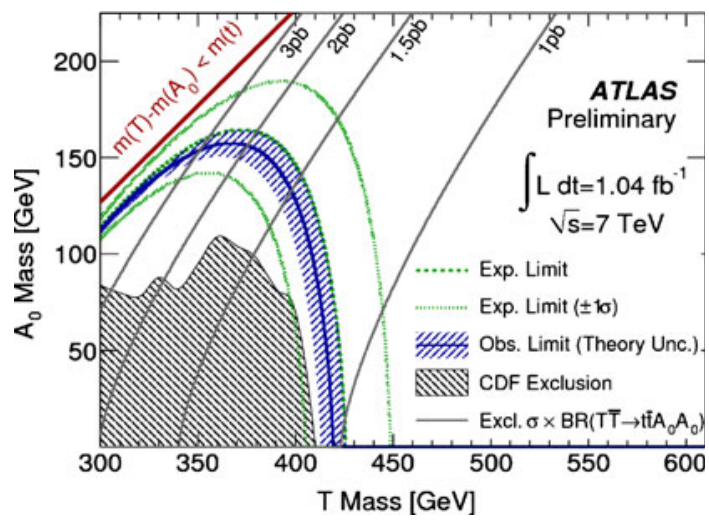

Figure 14. Exclusion limits on $A_{0}$ and $T$ from $T \rightarrow t A_{0}$ decays where the $A_{0}$ escapes detection, resulting from a search for top pair production with anomalous missing transverse momentum. The shaded area is the exclusion by CDF for this channel. 


\section{Top quark production}

ATLAS made a search for high mass top-like signatures in a dilepton final state and put limits on the heavy $Q_{4}$ for masses up to $270 \mathrm{GeV}$ at $95 \%$ CL [47], for $35 \mathrm{pb}^{-1}$.

Finally, special decays have been studied, such as the decays of top-like quarks decaying in $t Z$ as studied by CMS [48]: no $T \rightarrow t Z$ decays with masses below $417 \mathrm{GeV}$ are found at $95 \% \mathrm{CL}$. ATLAS searched for top-antitop pairs in association with anomalous missing transverse momentum in the lepton+jets $t t$ final state. Such an analysis is sensitive to possible $t A_{0}$ production with $A_{0}$ a long-lived neutral or invisible decaying particle. No such decays are found with a mass below $420 \mathrm{GeV}$ at $95 \%$ CL [49]. The results are shown in figure 14, and extend beyond the region so far covered by the CDF results.

In all, unfortunately so far no new signs of physics have been found in the top quark production channels. But the experiments will for sure keep up the searches in the top channels.

\section{Summary}

These are exciting times for top quark physics studies. The Tevatron will collect its final sample, and will have about $12 \mathrm{fb}^{-1}$ of data available for analyses. LHC is already up for precision measurements in the top sector. The total cross-sections are now measured with a precision of upto $7 \%$ at both the LHC and Tevatron. Single top quark production is now well established in the $t$-channel. The $s$ - and $W t$-channel analyses are in progress at the LHC, and the experiments should observe these in the present data run which extends till the end 2012.

The top quark is also an active area for searches for new physics. A tantalizing effect is seen in the forward-backward asymmetry and needs further study and likely more input from theory. In all there is plenty to do in the next years with the top quark samples collected at the Tevatron and LHC.

\section{References}

[1] CDF Collaboration: F Abe et al, Phys. Rev. Lett. 74, 2626 (1995)

[2] D0 Collaboration: S Abachi et al, Phys. Rev. Lett. 74, 2632 (1995)

[3] CDF Collaboration: T Aaltonen et al, Phys. Rev. Lett. 103, 92002 (2009)

[4] CDF Collaboration: V M Abazov et al, Phys. Rev. Lett. 103, 92001 (2009)

[5] G Tonelli, Proceedings of the ICHEP2010 (Paris, France, 2010)

[6] F Gianotti, Proceedings of ICHEP2010 (Paris, France, 2010)

[7] U Langenfeld, S Moch and P Uwer, Phys. Rev. D80, 054009 (2009) N Kidonakis, Phys. Rev. D82, 114030 (2010)

V Ahrens et al, J. High Energy Phys. 1009, 097 (2010)

V Ahrens et al, Phys. Lett. B703, 135 (2011)

[8] M Aliev et al, Comput. Phys. Commun. 182, 1034 (2011), arXiv:1007.1327

[9] D0 Collaboration, arXiv:1101.0124

[10] D0 Collaboration, arXiv:1107.5384

[11] CDF Collaboration, Note 10562

[12] CDF Collaboration, Note 10163

[13] ATLAS-CONF-2011-100

[14] CMS-TOP-11-007 
[15] F Deliot, arXiv:1111.6274

[16] ATLAS-CONF-2011-119

[17] ATLAS-CONF-2011-121

[18] CMS-TOP-11-005

[19] J Rojo, private ommunication

[20] R Thorne and G Watt, arXiv:1101.0536

[21] G Watt, arXiv:1106.5788

[22] S Moch and P Uwer, Phys. Rev. D80, 054009 (2009)

[23] D0 Collaboration, arXiv:1104.2887

[24] ATLAS-CONF-2011-054

[25] D0 Collaboration, arXiv: 1105.2788

[26] D0 Collaboration, arXiv:1108.3091

[27] CMS Collaboration, arXiv: 1106.3052

[28] ATLAS-CONF-2011-101

[29] ATLAS-CONF-2011-118

[30] ATLAS-CONF-2011-104

[31] CDF Collaboration, Phys. Rev. D83, 112003 (2011)

[32] D0 Collaboration, arXiv:1107.4995

[33] CMS-TOP-11-014

[34] ATLAS-CONF-2011-106

[35] CMS-EXO-11-065

[36] CDF Collaboration, arXiv:1107.5063

[37] CMS-TOP-10-007

[38] CMS-EXO-11-055

[39] ATLAS-CONF-2011-123

[40] CMS-EXO-11-006

[41] ATLAS-CONF-2011-87

[42] CMS-EXO-11-051

[43] CMS-EXO-11-050

[44] CDF Note 10395

[45] D0 Collaboration, arXiv:1104.4521

[46] CMS-EXO-11-006

[47] ATLAS-CONF-2011-023

[48] CMS-EXO-11-005

[49] ATLAS-CONF-2011-036 